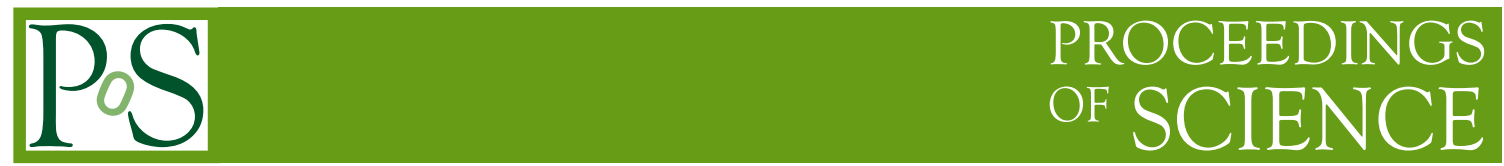

\title{
Production cross section of B-meson in ATLAS
}

\author{
E. Rossi* \\ On behalf of the ATLAS Collaboration \\ Sapienza - Università di Roma \\ E-mail: elvira.rossiecern.ch; ellyena.infn.it
}

\begin{abstract}
A new measurement of the production cross section of $B^{+}$as a function of transverse momentum $p_{T}$ and rapidity $y$ in proton-proton collisions at $\sqrt{s}=7 \mathrm{TeV}$, using $2.4 \mathrm{fb}^{-1}$ of data recorded with the ATLAS detector at the Large Hadron Collider is presented. The double differential cross section is measured over the range $|y|<2.25$ and $9<p_{T}<120 \mathrm{GeV}$, significantly wider than in previous measurements. Comparisons with next-to-leading order theoretical predictions (POWHEG+PYTHIA, MC@NLO+HERWIG and FONLL) are discussed.
\end{abstract}

XXI International Workshop on Deep-Inelastic Scattering and Related Subject -DIS2013, 22-26 April 2013

Marseilles, France

${ }^{*}$ Speaker. 


\section{Introduction}

Data obtained by the Large Hadron Collider collaborations provide a chance for measurement of $b$-production in a new energy regime, at higher transverse momenta $\left(p_{T}\right)$ and in wider rapidity (y) ranges than have previously been studied. Testing next-to-leading order (NLO) calculations of Quantum Chromodynamics (QCD) is important as there are large theoretical uncertainties deriving from renormalization and factorization scales. A measurement of the exclusive $B^{+}$production cross-section in the decay channel $B^{+} \rightarrow J / \psi K^{+} \rightarrow \mu^{+} \mu^{-} K^{+}$, as a function of $B^{+}$transverse momentum and rapidity, is presented [1]. This analysis uses $2.4 \mathrm{fb}^{-1}$ of data recorded by the ATLAS detector in pp collisions at $\sqrt{s}=7 \mathrm{TeV}$ and extends the measurement of the $B^{+}$cross section up to $p_{T}<120 \mathrm{GeV}$, allowing comparisons with NLO predictions in four rapidity regions in the range $|y|<2.25$.

\section{The ATLAS Detector}

The ATLAS experiment uses a general purpose detector consisting of an inner tracker, a calorimeter and a muon spectrometer. The inner detector (ID) directly surrounds the interaction point; it includes a silicon pixel detector, a silicon strip detector and a transition radiation tracker, and is embedded in an solenoidal $2 \mathrm{~T}$ magnetic field. The ID $(|\eta|<2.5)$ is enclosed by a calorimeter system containing electromagnetic and hadronic sections. The calorimeter is surrounded by a large muon spectrometer inside an air-core toroid magnet system which contains a combination of monitored drift tubes and cathode strip chambers, designed to provide precise position measurements in the bending plane. In addition, resistive plate chambers and thin gap chambers with a fast response time are used primarily to trigger muons. The ATLAS trigger system has three levels: the hardware-based Level-1 trigger and the two stage High Level Trigger (Level-2 trigger and Event Filter). Details on the ATLAS detector and trigger system can be found in [2].

\section{Event selection}

Data for this analysis were collected during the 2011 using a di-muon trigger that requires the presence of two muon candidates with opposite charges that are consistent with having a common vertex and have $p_{T}>4 \mathrm{GeV}$ and $|\eta|<2.3$. Two Monte Carlo (MC) samples have been used in this analysis: the first simulates the signal decay $B^{+} \rightarrow J / \psi K^{+} \rightarrow \mu^{+} \mu^{-} K^{+}$while the second simulates the inclusive $b \bar{b} \rightarrow J / \psi X \rightarrow \mu^{+} \mu^{-} X$. Both samples were generated with Pythia6 [3] using the ATLAS MC11 tune [4]. The ATLAS detector and its response were simulated using GEANT4 [5] and fully reconstructed with the same software that is used to process the data. Reconstructed muons are required to have a track reconstructed in the MS matched with a track reconstructed in the ID and their kinematic properties are determined from the parameters of the ID tracks. Muon and kaon candidates tracks are required to pass quality cuts to ensure accurate ID measurements. Events are required to contain at least one pair of reconstructed muons fitting successfully to a common vertex. The muon pairs with invariant mass in the range $2.7-3.5 \mathrm{GeV}$ are considered as $J / \psi \rightarrow \mu^{+} \mu^{-}$decay candidates. The muon tracks of the selected $J / \psi$ candidates are again fitted to a common vertex with an additional third track of $p_{T}>1 \mathrm{GeV}$. The three-track vertex fit is 


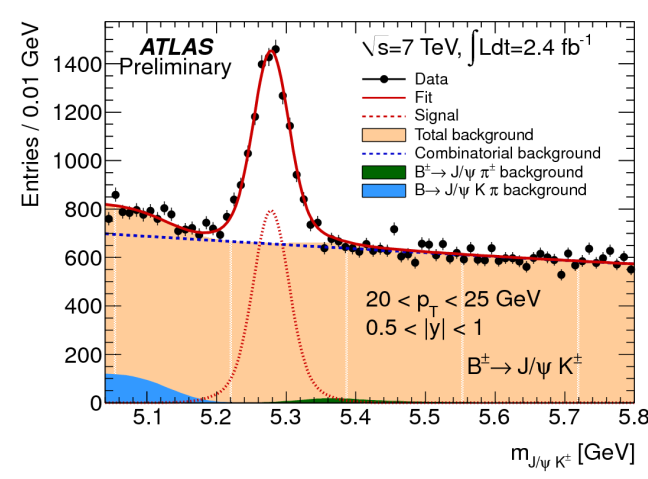

(a)

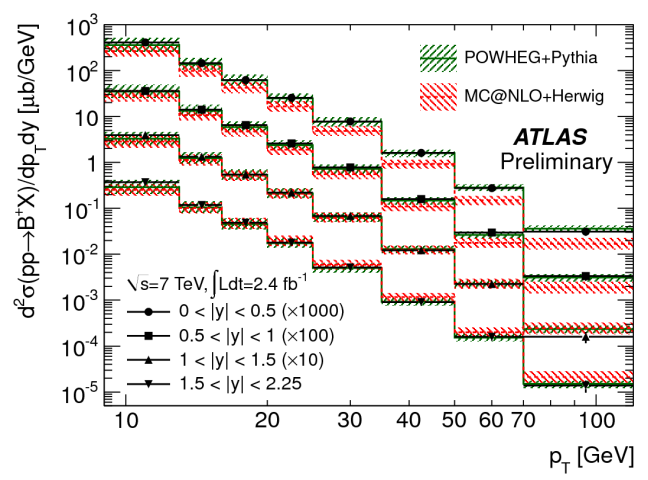

(b)

Figure 1: a) Invariant mass of $B^{ \pm}$candidates in an intermediate $p_{T}$ interval in a central rapidity region [1]. The points are data and the solid line is the result of the fit. b) Double-differential cross section of $B^{+}$production as a function of $p_{T}$ for different rapidity ranges [1]. The points correspond to data with the combination of the statistical and systematic uncertainty.

performed by constraining the muon tracks to the $J / \psi$ world average mass [6] and assigning kaon mass to the third track. The $B^{ \pm}$candidates are retained in the mass range $5.04-5.8 \mathrm{GeV}$ with $p_{T}>9 \mathrm{GeV}$ and $|y|<2.3$.

\section{4. $B^{+}$differential production cross-section measurement}

The measurement of the differential cross-section is determined as:

$$
\frac{d^{2} \sigma\left(p p \rightarrow B^{+} X\right)}{d p_{T} d y}=\frac{N^{B^{+}}}{\mathscr{L} \cdot \mathscr{B} \cdot \Delta p_{T} \cdot \Delta y}
$$

where $N^{B^{+}}$is the $B^{+} \rightarrow J / \psi K^{+}$signal decays produced, $\mathscr{L}$ is the integrated luminosity of the data sample, $\mathscr{B}$ is the total branching ratio of the signal decay $(6.03 \pm 0.21) \times 10^{-5}$ obtained combining the world average values of the branching ratios for $B^{+} \rightarrow J / \psi K^{+}$and $J / \psi \rightarrow \mu^{+} \mu^{-}$and $\Delta p_{T}$ and $\Delta y$ are the $p_{T}$ and rapidity bin widths. To increase the statistical precision both $B^{+}$and $B^{-}$ reconstructed decays are used and $N^{B^{+}}$is derived from the average yield of the two states after correcting for detector effects and acceptance: $N^{B^{+}}=\frac{1}{A} \frac{N_{\text {reco }}^{B^{ \pm}}}{\varepsilon^{B^{+}}+\varepsilon^{B^{-}}}$where $N_{\text {reco }}^{B^{ \pm}}$is the number of reconstructed signal events, $A$ is the acceptance and $\varepsilon^{B^{+/-}}$are the efficiencies for $B^{+/-}$. The overall efficiency for a $B^{+} \rightarrow J / \psi(\mu \mu) K^{+}$decay within a $p_{T}-y$ bin is obtained from MC simulations and it's defined as the ratio of the number of signal events generated and the number of reconstructed events passing all the offline selection requirements in that bin.

The number of reconstructed $B^{ \pm}$mesons is obtained using a binned maximum likelihood fit to the invariant mass of the selected candidates. The invariant mass shape of signal and background is studied in each $p_{T}-y$ bin using MC in order to extract the fit models. The probability density function (pdf) for the signal is defined as the sum of two Gaussians of relative fraction $f_{1}$ and corresponding widths $\sigma_{1}, \sigma_{2}$, both centered at the reconstructed mass of $B^{ \pm}$candidates. There are three sources of background to the signal process: 

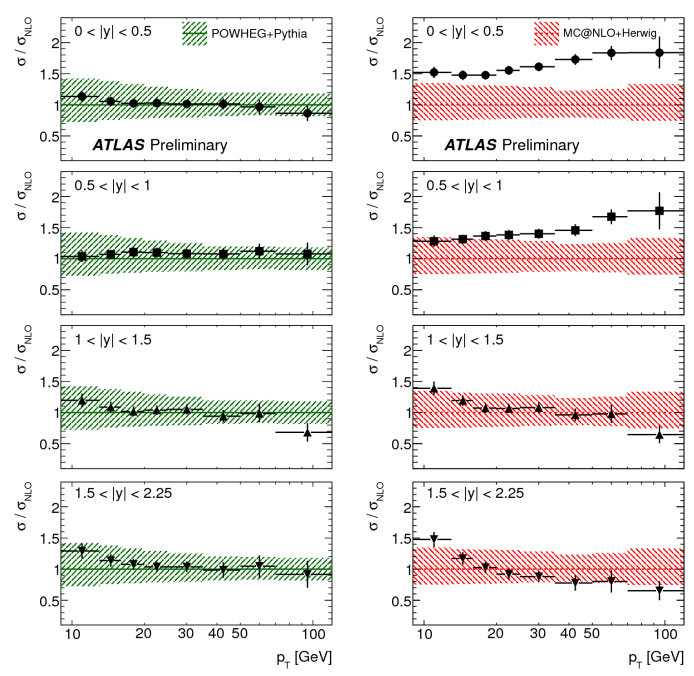

Figure 2: Ratio of the measured cross section to the theory predictions of Powheg (left) and MC@NLO (right) in four rapidity regions [1]. The points correspond to data with the combination of the statistical and systematic uncertainty.

- $B^{+} \rightarrow J / \psi \pi^{+}$in which the kaon mass is wrongly assigned to the pion (modeled with a Crystal Ball function);

- $B^{ \pm / 0} \rightarrow J / \psi K^{* \pm / 0} \rightarrow J / \psi K \pi$ and $B^{ \pm / 0} \rightarrow J / \psi K^{ \pm} \pi^{0 / \mp}$ where the final state pion is not associated to a decay vertex, creating a resonance structure approximately one pion mass away from the $B^{ \pm}$mass;

- the contributions that are not peaking in mass come from random combinations of $J / \psi$ with a track.

The extraction of the signal yield is done in two steps. First, the shapes of the signal and background pdf's are obtained from fits to MC for each $p_{T}-y$ interval and then the data are fitted in the same $p_{T}-y$ interval. The parameters for the shape of the signal pdf and the resonant background are fixed to the results of the fits to MC. The relative normalisation of the $B^{ \pm} \rightarrow J / \psi \pi^{ \pm}$decay to the signal is fixed to the fraction of the world averaged values for their branching ratios, and is corrected for the difference in acceptance of the two decay modes. The reconstructed mass $m_{B^{ \pm}}$is obtained from data by fitting the invariant mass of the selected candidates, and is fixed throughout the fits in $p_{T}$ intervals. Therefore, when fitting the data in each $p_{T}-y$ interval, the free parameters are the normalisation of the signal and the partially reconstructed B-decays, and the slope of the combinatorial background. The results of the fits to the invariant mass of the selected $B^{ \pm}$candidates from data are exemplified in Figure 1 plot a) for a region of intermediate $p_{T}$ and of central rapidity. The total number of signal $B^{ \pm}$events observed in data in the full $p_{T}$ and $y$ range before acceptance and efficiency corrections is $125600 \pm 1201$, where the quoted uncertainty is statistical. These events are split in four intervals of $|y|$ and eight intervals of $p_{T}$ for the differential cross section measurement. 


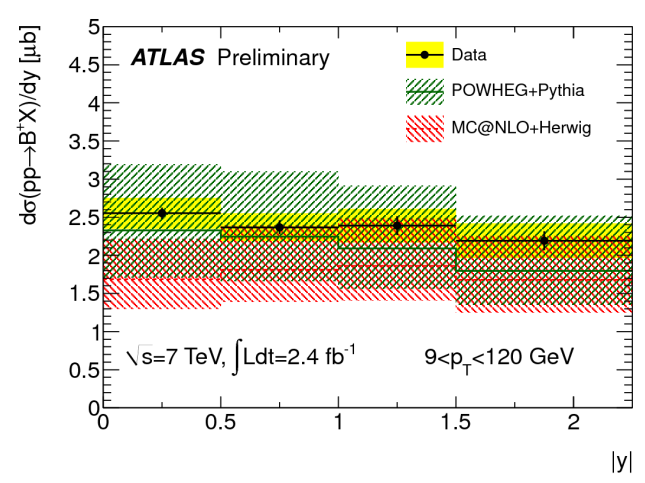

(a)

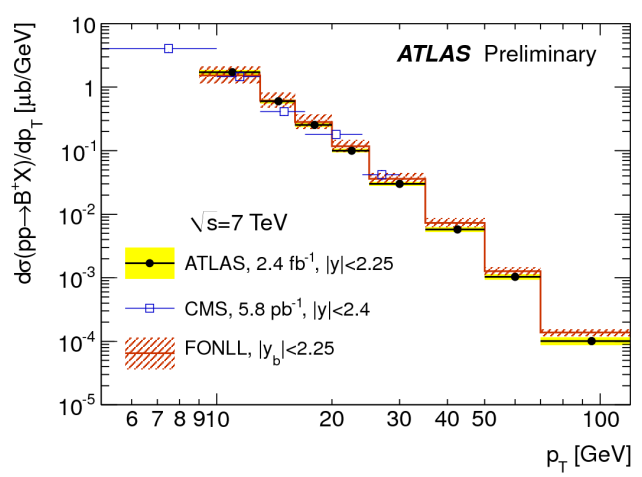

(b)

Figure 3: Differential cross section of $B^{+}$production as a function of rapidity (plot a) and $p_{T}$ (plot b) [1]. In both plots points are data with statistical uncertainty and the yellow rectangles show the combined statistical and systematic uncertainty.

The differential cross section for $B^{+}$production is obtained, and is shown in Figure 1 plot b) as a function of $p_{T}$ of the $B^{+}$meson in four rapidity regions. Each point includes the combined statistical and systematic uncertainty. The results are compared to NLO predictions obtained with Powheg(+Pythia) and MC@NLO(+Herwig). The NLO predictions are assigned an uncertainty due to renormalisation and factorisation scale and the b-quark mass, shown with a hatched area. To allow for a better comparison between the measured cross section and the NLO predictions, Figure 2 shows its ratio for each rapidity region separately for Powheg and MC@NLO. The double differential cross section is summed over the $p_{T}$ intervals, or over the rapidity ranges, in order to obtain the differential cross section, shown Figure 3. The measurement is compared to the Powheg and MC@NLO predictions (Figure 3 plot a) and to FONLL calculations and to CMS results [7] (Figure 3 plot b). The theoretical uncertainty includes the uncertainties on the b-quark mass, renormalisation and factorisation scales. The measured differential cross section is compared with the predictions of NLO QCD computations. The predictions are obtained using Powheg+Pythia and MC@NLO+Herwig and are quoted with an uncertainty from renormalisation and factorisation scales and b-quark mass of the order of $20 \%-40 \%$. Within these uncertainties, Powheg+Pythia is altogether in agreement with the measurement in absolute scale and in the dependences on $\mathrm{pT}$ and y. At low $|y|$, MC@NLO+Herwig predicts lower production cross section and a softer $p_{T}$ spectrum than the one observed in data, which becomes harder for $|y|>1$. An FONLL calculation, assuming a hadronisation fraction of $f_{b} \rightarrow B^{+}$of $(40.1 \pm 1.3) \%$ [6] to fix the overall scale, is in good agreement with the measurement of $d \sigma / d p_{T}$, in particular for $p_{T}<30 \mathrm{GeV}$.

\section{References}

[1] ATLAS Collaboration, ATLAS-CONF-2013-008 (https://cds.cern.ch/record/1522478).

[2] ATLAS Collaboration, JINST 3 (2008) S08003; ATLAS Collaboration, Eur. Phys. J. C72 (2012) 1849, arXiv:1110.1530 [hep-ex]. 
[3] T. Sjostrand, S. Mrenna, and P. Z. Skands, JHEP 0605, 026 (2006), arXiv:hep-ph/0603175 (http://arxiv.org/abs/hep-ph/0603175).

[4] ATLAS Collaboration, ATL-PHYS-PUB-2011-009, http://cdsweb.cern.ch/record/1363300.

[5] S. Agostinelli et al., Nucl. Instrum. Meth. A 506, 250 (2003); ATLAS Collaboration, Eur. Phys. J. C 70, 823 (2010), arXiv:1005.4568 [physics.ins-det].

[6] J. Beringer et al. (Particle Data Group), Phys. Rev. D86, 010001 (2012).

[7] CMS Collaboration, Phys. Rev. Lett. 106 (2011) 112001, arXiv:1101.0131 [hep-ex]. 